\title{
丙酮参与偶联反应研究的新进展
}

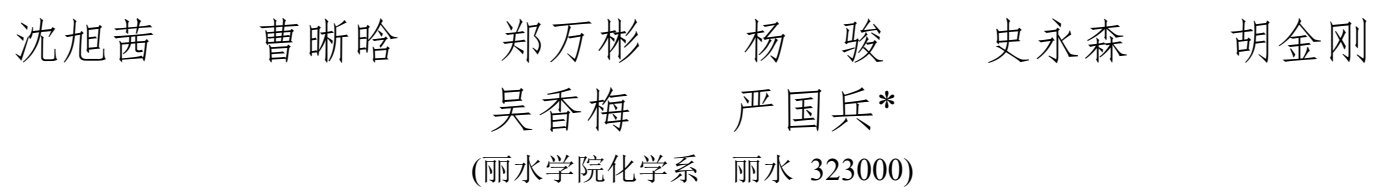

\begin{abstract}
摘要 丙酮是一种最简单的酮类化合物, 具有酮类的典型反应. 综述了近年来丙酮参与偶联反应的研究进展, 主要包 括碳-碳、碳-杂的形成及其反应机理的探讨.
\end{abstract}

关键词 丙酮; 偶联反应; 反应机理

\section{Recent Progress in the Research of Acetone in Coupling Reactions}

\author{
Shen, Xuqian Cao, Xihan Zheng, Wanbin Yang, Jun Shi, Yongsen Hu, Jingang \\ Wu, Xiangmei Yan, Guobing* \\ (Department of Chemistry, Lishui University, Lishui 323000)
}

\begin{abstract}
Acetone is the simplest ketone, which has the typical reaction of ketones. In this paper, the recent development centering the coupling reactions of acetone including the formation of carbon-carbon, carbon-heteroatom bonds and the discussion of reaction mechanism, is reviewed.
\end{abstract}

Keywords acetone; coupling reaction; reaction mechanism

丙酮是一种非常重要的有机化工原料, 广泛应用于 化学工业生产. 一方面, 丙酮作为溶剂主要用于炸药、 塑料、橡胶、纤维、制革、油脂、喷漆等行业; 另一方 面, 它也作为合成环氧树脂、聚碳酸酯、甲基丙烯酸甲 酯、聚异戊二烯橡胶等重要化工原料. 丙酮是一种最简 单的酮类化合物, 具有酮的典型反应, 例如亲核一加成 反应、 $\alpha$-氢的反应、催化氢化反应等. 这些反应在有机 合成中的应用非常广泛. 在过去的几十年, 有大量文献 报道以丙酮为原料, 利用这些经典的反应合成了各式各 样的目标分子 ${ }^{[1]}$. 探索酮类化合物的新反应是有机化学 工作者长期追求的目标. 近年来, 丙酮参与过渡金属催 化的交叉偶联反应取得了一些突破性的进展; 另外, 丙 酮参与自由基的氧化偶联构建碳一碳、碳一杂键也有相关 文献报道. 因此, 本文综述了丙酮参与偶联反应的最新 进展及其反应机理的探讨.

\section{1 过渡金属催化的偶联反应}

过渡金属催化的交叉偶联反应是现代有机合成的
重要工具, 利用此类反应可高效、高选择性地构建 碳碳及碳-杂键等化学键 ${ }^{[2]} .1997$ 年, Buchwald 和 Hartwig 小组 ${ }^{[3]}$ 同时报道了在碱性条件下钯催化的羰基 $\alpha$-位芳基 化反应. 这种开创性的工作引领了国内外有机化学工作 者对该类催化体系进行了广泛而深入的研究. 随后, 国 内外也有相应的文献[4]对钯催化羰基衍生物 $\alpha$-位芳基 化反应的研究进展进行讨论和论述. 尽管该研究领域已 经取得了重大的进展，但要实现一些简单酮类化合物的 单芳基化反应还存在一定的挑战性.

丙酮是一种最简单的酮类化合物, 结构也对称. 实 现丙酮羰基 $\alpha$-位的单芳基化的困难在于：一方面，以这 种最简单酮为底物, 反应过程中经过了芳基-钯(II)-烯 酮的关键中间体，还原消除特别慢；而且非常困难；另 一方面，单芳基化产物的羰基 $\alpha$-氢酸性增强了，还可以 进一步发生芳基化，导致多芳基化的混合产物. 早期的 文献报道了利用钯催化丙酮的烯醇硅醚等类似物与卤 代芳烃反应，能够实现丙酮羰基 $\alpha$-位的单芳基化 ${ }^{[5]}(\mathrm{Eq}$. 1). 然而, 这些反应需要预先合成烯醇醚的底物, 且以

\footnotetext{
* Corresponding author. E-mail: gbyan@1su.edu.cn

Received July 28, 2016; revised September 15, 2016; published online October 9, 2016.

Project supported by the National Natural Science Foundationof China (No. 21572094).

国家自然科学基金(No. 21572094)资助项目.
} 
当量的锡试剂或锌试剂为添加剂, 不符合原子经济性发 展的理念.

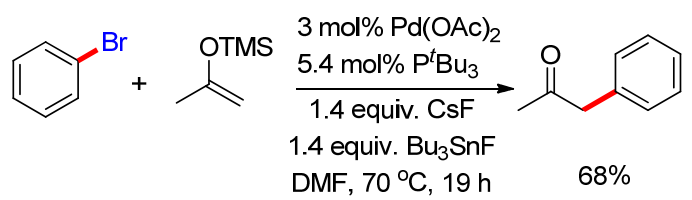

2011 年, Stradiotto 课题组 ${ }^{[6]}$ 在该研究领域取得了突 破性进展. 他们将一种新颖的 P,N-配体应用到钯催化丙 酮与芳香卤化物的芳基化反应体系, 能够很好地控制反 应活性及选择性, 实现了丙酮羰基 $\alpha$-位高选择性的单芳 基化(Eq. 2). 一系列富电子及缺电子的芳基氯、澳、碘 化物都适用于该反应. 从反应的结果来看, 富电子底物 的反应活性较高, 且产率也相对较高. 除了芳基卤代物 外, 富电子及位阻较大的对甲基苯磺酸苯酚酯衍生物在 该反应条件下也能提供较高的收率. 然而, 在篮选反应 条件的过程中, 他们也检测到少量的二芳基化产物. 催 化剂及碱的用量、丙酮与碱的比例、反应的时间等参数 对选择性有一定的影响, 但该催化体系的配体对选择性 单芳基化起着至关重要的作用. 该反应充分利用了一种 简单、廉价易得的三一碳原子的化工原料, 不需预先合成 烯醇醚化合物. 另外, 该反应还具有催化剂用量少、反 应条件温和、官能团耐受性好及底物的适用范围广等优 点. 此后, 他们利用该催化体系, 以磷酸钾为碱, 叔丁 醇及 1,4 -二氧六环为共溶剂, 实现了甲磺酸苯酚酯衍生 物与丙酮的偶联反应 ${ }^{[7]}$.

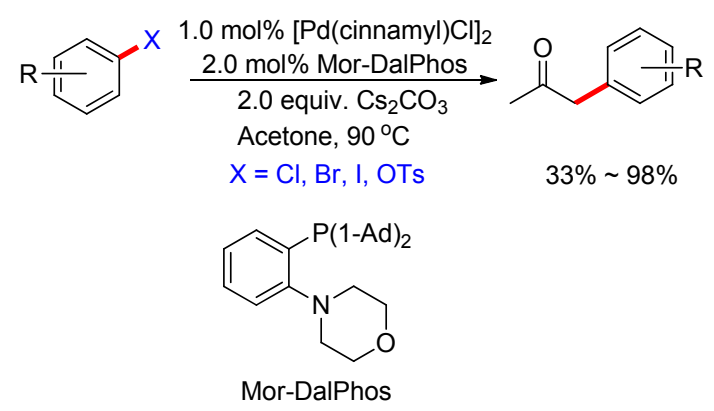

他们课题组还将该方法学应用于 “一锅法” 的多组 分反应合成多官能团化的吲哚衍生物(Eq. 3) ${ }^{[8]}$. 该反应 是通过经典的 Buchwald-Hartwig 胺化反应、丙酮的单 芳基化反应及缩合反应三步串联反应完成的.

这种引领性工作促使国内外有机化学工作者探 索了一系列配体在钯催化丙酮羰基 $\alpha$-位芳基化反应体 系中的应用. 2012 年, Ackermann 课题组 ${ }^{[9]}$ 报道了以 XantPhos 为配体, 利用醋酸钯催化丙酮与咪唑基磺酸酯 的偶联反应, 实现了丙酮羰基 $\alpha$-位单芳基化(Eq. 4). 在 此反应条件下，他们还探索了不同磺酸酯分子间的竞争

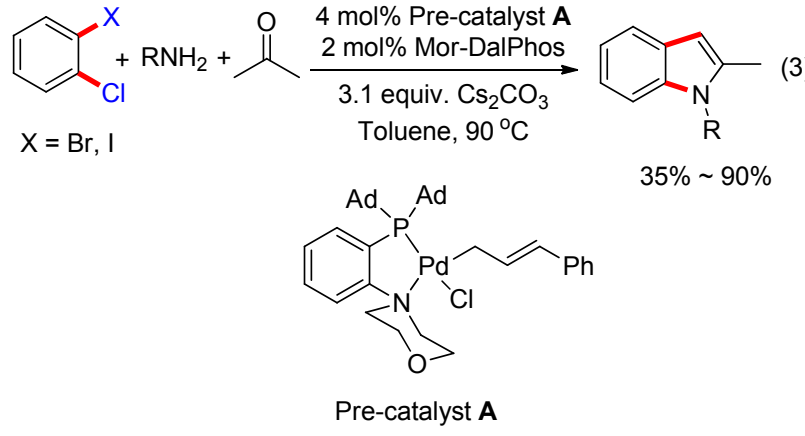

反应，实验结果表明咪唑基磺酸酯的反应活性比对甲苯 磺酸酯、甲磺酸酯高, 而且只得到咪唑基磺酸酯与丙酮 偶联的单一产物. 该反应优点是醋酸钯催化剂和双膦配 体相对便宜.

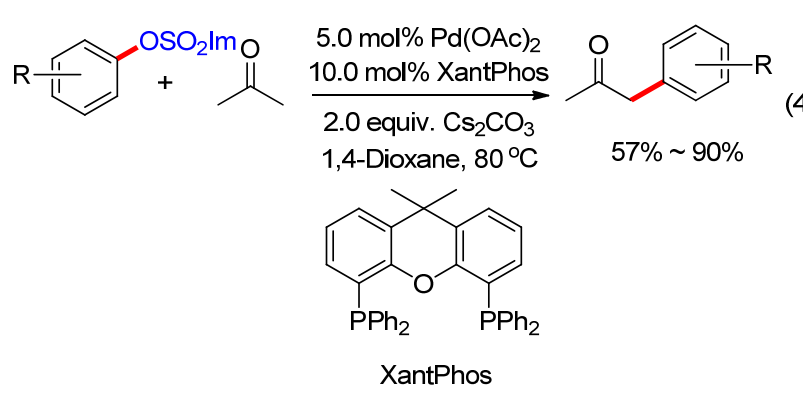

2013 年, Ma 课题组 ${ }^{[10]}$ 发展了一种新的 P,N-配体 (Zheda-Phos), 并应用于钯催化丙酮羰基 $\alpha$-位芳基化反 应体系(Eq. 5). 他们提出了可能的反应机理(Scheme 1): 首先，钯催化剂前体/Zheda-Phos 与卤代芳烃发生氧化 加成, 得到钯(II)的中间体 $\mathbf{A}$; 在碱的存在下, 这种二价 钯的中间体被丙酮烯醇式化合物亲核进攻，产生中间体 $\mathbf{B}$; 由于中间体 $\mathbf{B}$ 位阻大，不太稳定，很快会发生还原 消除得到单芳基化产物. 该配体中的甲氧基在反应过程 中起着非常重要的作用. 它的给电子性能有利于氯代芳 烃的氧化加成过程, 它的空间位阻又能极大地促进钯 (II)中间体的还原消除。该反应底物的适用范围广，一 系列含氮、硫等杂环氯代芳烃都能获得较高的收率.

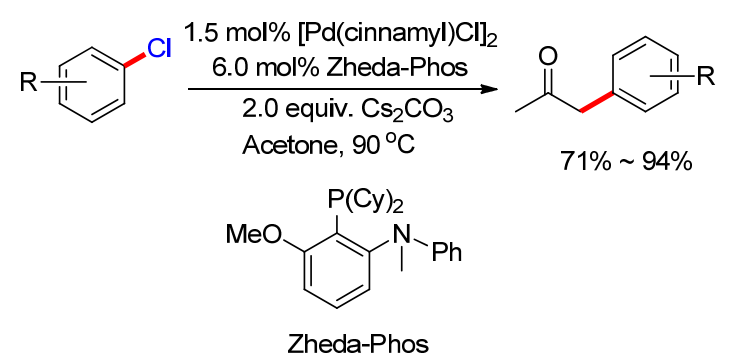

(5)

在过去的几十年, 含二茂铁的富电子、大位阻的膦 配体被广泛应用于钯催化的交叉偶联反应来构建碳一碳 键 $^{[11]} .2014$ 年, Lang 课题组 ${ }^{[12]}$ 发展了一种在空气条件下 


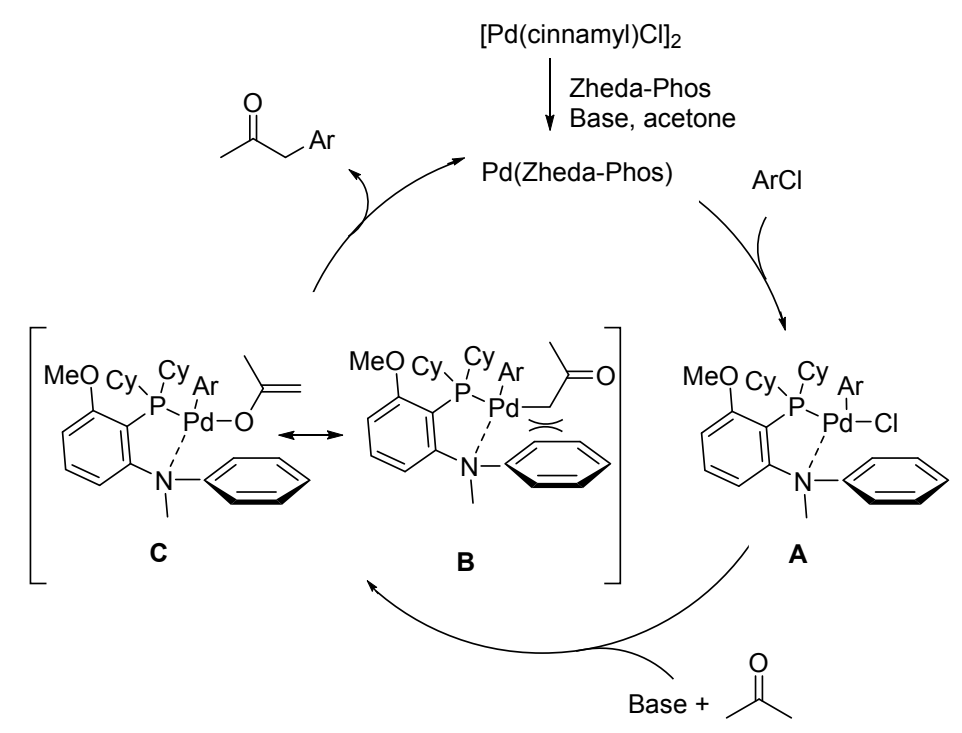

图式 1 可能的反应机理

Scheme 1 Possible mechanism

稳定的二茂铁 P,N-配体, 能够很好地应用于钯催化丙酮 的单芳基化反应(Eq. 6). 与之前报道的反应相比，该催 化体系还可以适用于缺电子的、大位阻的卤代芳烃及杂 环芳烃. 对于大部分反应而言, 钯催化剂的用量可以降 低到 $0.5 \mathrm{~mol} \%$, 产率还能达到 $95 \%$ 以上, 说明该催化体 系的催化效果的确不错.
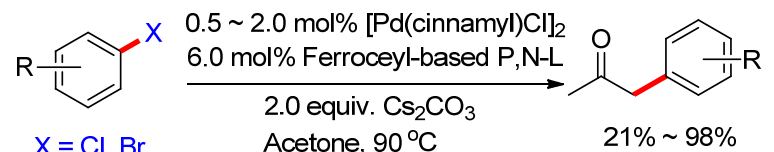

(6)

$\mathrm{X}=\mathrm{Cl}, \mathrm{Br}$

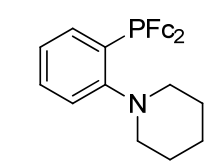

Ferroceyl-based P,N-L

随后, Stradiotto 课题组 ${ }^{[13]}$ 第一次报道了在室温条件 下, 以二茂铁的双膦配体与钯为催化体系, 实现了丙酮 羰基 $\alpha$-位单芳基化反应(Eq. 7). 该反应的底物适用范围 很广, 一系列富电子和缺电子的氯、澳、碘代芳烃及杂 环芳烃都能给出较高的收率.

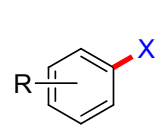

$3.75 \mathrm{~mol} \%\left[\mathrm{Pd}(\text { cinnamyl)Cl}]_{2}\right.$ Pd:JosiPhos (1:1.15)

$\mathrm{X}=\mathrm{Cl}, \mathrm{Br}, \mathrm{I}$

Acetone, $25^{\circ} \mathrm{C}, 48 \mathrm{~h}$
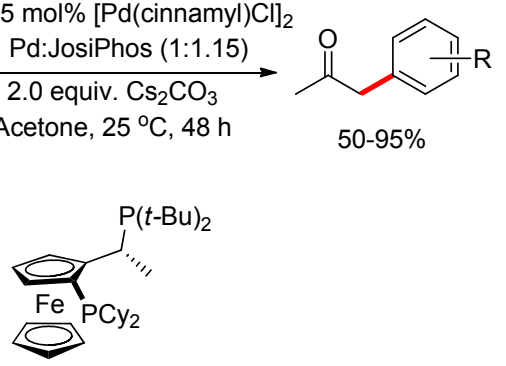

2015 年, Kwong 课题组 ${ }^{[14]}$ 发展了一种富电子的、大 位阻的、含吲哚基的单膦配体，应用于该类芳基化反应 (Eq. 8). 该配体中的两个甲氧基对该反应的催化性能起 着非常重要的作用, 既有利于碳一氯键的氧化加成, 又 有利于钯中间体的还原消除. 对于大部分底物而言, 醋 酸钯催化剂的用量为 $0.5 \mathrm{~mol} \%$ 时都能给出较高的收率, 有的甚至可以降低到 $0.1 \mathrm{~mol} \%$ ，也不会影响产物的收 率.
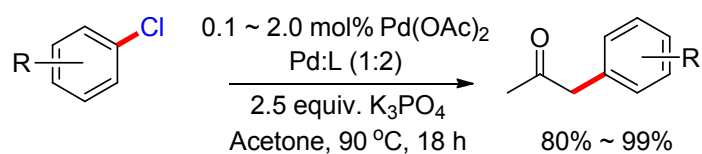

$80 \% \sim 99 \%$<smiles>COc1cccc(OC)c1-c1c(-c2ccccc2)c2ccccc2n1C</smiles>

Indolylphosphine ligand
Beller 课题组 ${ }^{[15]}$ 发展了钯催化的碘代芳烃、一氧化 碳及丙酮的三组分反应，有效地合成了一系列的 1,3-二 羰基化合物(Eq. 9). 该配体在催化体系中起着多方面的 作用, 既有助于芳基碘化物的氧化加成, 又能适合于一 氧化碳的插入, 还能促进钯中间体的还原消除. 该反应 的底物适用范围较广, 富电子及缺电子取代的芳烃及杂 环芳烃碘化物都能够给出中等以上的收率. 然而, 在反 应的过程中, 他们还监测到苯甲酸及苯甲醛等少量的副 产物. 这些结果表明: 可能是丙酮烯醇化合物的亲核钯 化过程相对较慢导致的. 


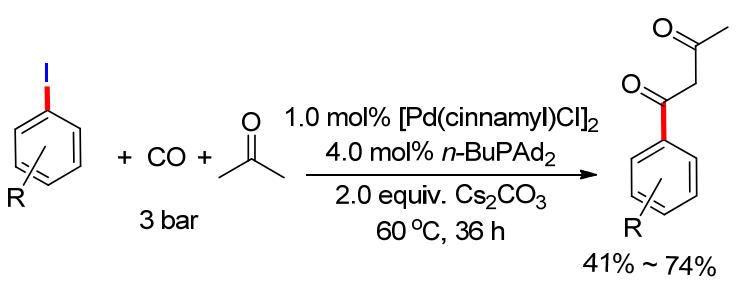

(9)

迄今为止, 在钯催化丙酮羰基 $\alpha$-位的单芳基化反应 体系中, 富电子、大位阻的膦配体对于该反应活性及选 择性非常关键. 尽管丙酮单芳基化产物的 $\alpha-\mathrm{H}$ 的酸性增 强了, 但同时它的位阻也增加了, 且所有的催化体系配 体的位阻较大，再发生芳基化非常困难，基本上得不到 双芳基化的产物.

\section{2 自由基氧化偶联反应}

自由基化学因其具有反应条件温和、高区域选择性 与立体选择性等独特的优点, 已经成为有机合成化学学 科的重要研究方向和前沿热点领域之一 ${ }^{[16]}$. 酮类化合 物在光化学研究领域引起人们极大的兴趣. 由于酮羰基 的氧和碳一氧双键分别有一对容易激发的孤对电子和 $\pi$ 电子, 在光照条件下受光激发后, 参与各种有机化学反 应，如 Norrish 类型的裂解反应、光氧化还原反应、加成 反应等. 早期丙酮参与光化学反应研究也有很多相关报 道 ${ }^{[17]}$. 由于篇幅有限, 我们只介绍一个具有代表性的例 子. 2008 年, Hirai 等 ${ }^{[17 i]}$ 报道了在光条件下, 水促进的烯 烃与丙酮的偶联反应，高效地合成了一系列甲基酮化合 物(Eq. 10). 该反应可能的机理: 在光条件下产生的丙酩 自由基对烯烃双键的加成反应(Scheme 2). 接下来, 我 们主要介绍近几年来丙酮参与自由基的氧化偶联反应.
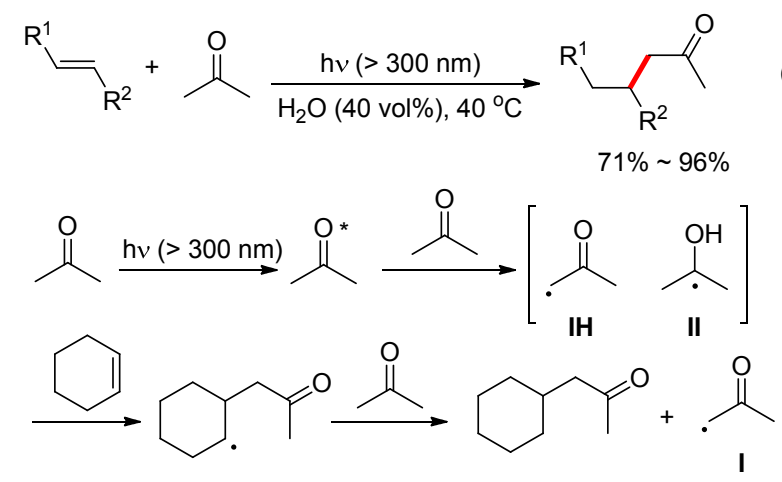

图式 2 可能的反应机理

Scheme 2 Possible mechanism

2014 年, Klussmann 课题组 ${ }^{[18]}$ 报道了有机酸催化酮 对烯烃的自由基氧化加成反应，得到了烷基化-过氧化 的烯烃双官能团化产物(Eq. 11). 尽管该反应底物的适 用范围较广, 但大部分反应只有中等的收率. 布朗斯特 酸在该反应中起着非常重要的作用, 即促使丙酮自由基
的产生(Scheme 3). 在强酸的存在下, 过氧叔丁醇对丙 酮的亲核一加成得到中间体 $\mathbf{A}$, 随后脱水生成烯基过氧 化物中间体 B. 这种烯基过氧化物很容易均裂产生共振 一稳定化的丙酮自由基与叔丁氧自由基，再发生后续的 烯烃自由基加成反应，得到双官能团化产物.

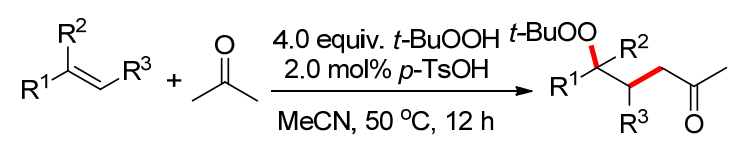

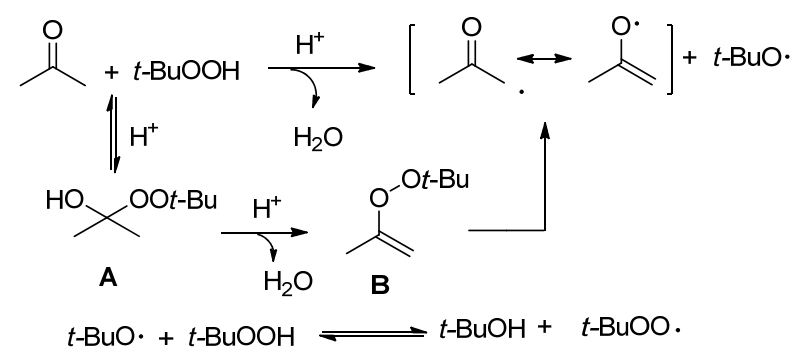

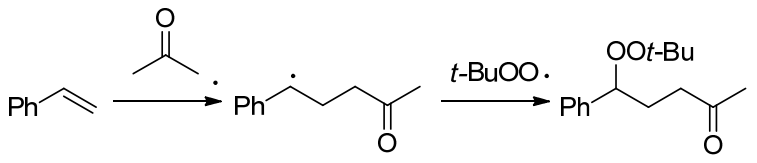

图式 3 可能的反应机理

Scheme 3 Possible mechanism

随后, Xia 和 Klussmann 课题组 ${ }^{[19]}$ 报道了有机酸催 化丙酮对芳基丙烯酰胺的自由基加成与环化的串联反 应, 合成了一系列氧化吲哚的衍生物(Eq. 12). 当反应底 物氮原子上基团为磺酸基或氢原子 $\left(\mathrm{R}^{2}=\mathrm{Ts}, \mathrm{H}\right)$ 时，则会 得到烷基化-过氧化的烯烃双官能团化产物.

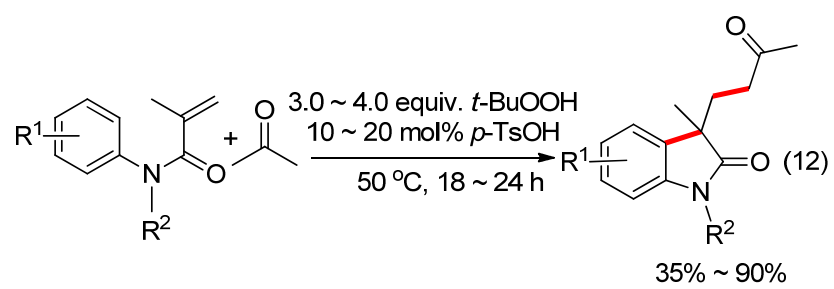

2014 年, $\mathrm{Ji}$ 课题组 ${ }^{[20]}$ 报道了 $\alpha, \alpha$-二芳基烯丙醇与简 单羰基化合物的烷基化反应(Eq. 13). 该反应底物的普 适性很广，一系列的羰基化合物都能给出较高的收率, 如脂肪䣶和环酮、酯和内酯、 $N$-甲基酰胺等. 另外，该 反应的条件非常简单，除了自由基的前体化合物外，不 需要任何添加剂. 该反应的机理经由了自由基对烯烃双 键的加成，以及分子内芳基的 1,2-迁移过程，得到 1,5二羰基化合物(Scheme 4). 芳基的 1,2-迁移受到了芳环 上取代基的电子及位阻效应的影响，一般来说，缺电子 的芳烃及位阻小的芳烃更容易迁移. 


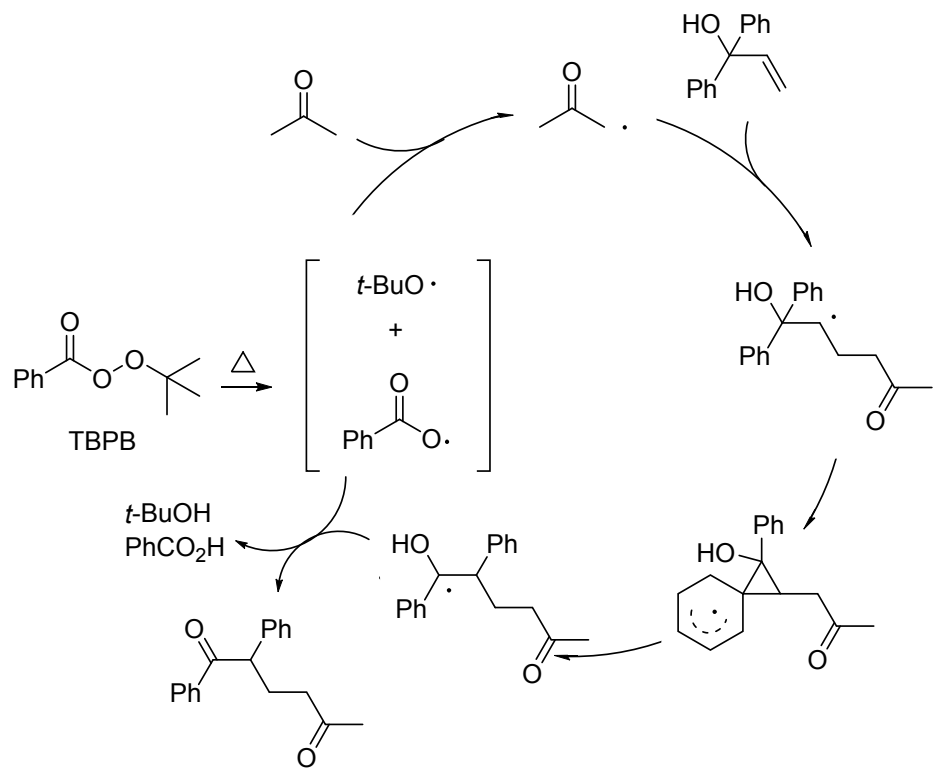

图式 4 可能的反应机理

Scheme 4 Possible mechanism

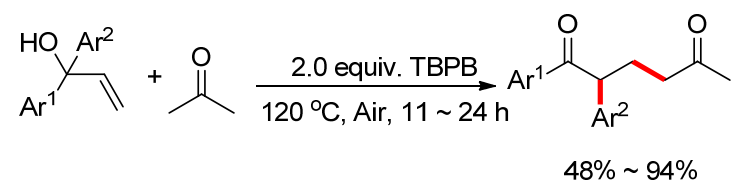

2014 年, $\mathrm{Li}$ 课题组 ${ }^{[21]}$ 报道了银催化烯烃不饱和键的 碳一氟双官能团化反应(Eq. 14). 该反应的底物适用范围 很广, 区域选择性好, 产物产率较高. 另外, 该反应还 可以放大到克级规模, 依然具有良好的催化效果, 展示 了该催化体系的实用性. 在 $\mathrm{Ag}(\mathrm{I})-$ SelectFluor 氟试剂的 氧化体系条件下, 丙酮能够生成丙酮基自由基, 再发生 后续的烯烃自由基加成反应, 得到双官能团化产物 (Scheme 5).

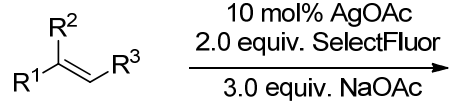

$$
\begin{aligned}
& \text { Acetone } / \mathrm{H}_{2} \mathrm{O}(\mathrm{V}: \mathrm{V}=1: 1) \\
& 50^{\circ} \mathrm{C}, 12 \mathrm{~h}
\end{aligned}
$$

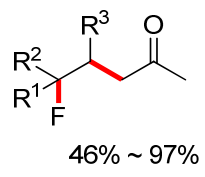

(14)

2015 年, Wang 课题组 ${ }^{[22]}$ 报道了在氧化条件下, 利 用 铜一锰双金属催化烯烃与丙酮的反应, 得到了 $1,4-$ 二 羰基化合物(Eq. 15). 尽管该反应的底物适用范围较宽, 区域选择性好, 但大部分产物的收率中等偏低, 而且反 应所需的时间较长. 该反应的机理类似于 Klussmann 课 题组报道烯烃的烷基化-过氧化双官能团化反应 ${ }^{[19]}$ ，只 不过这种双官能团化的产物在该反应体系条件下很容 进一步转化为 1,4 -二羰基化合物.

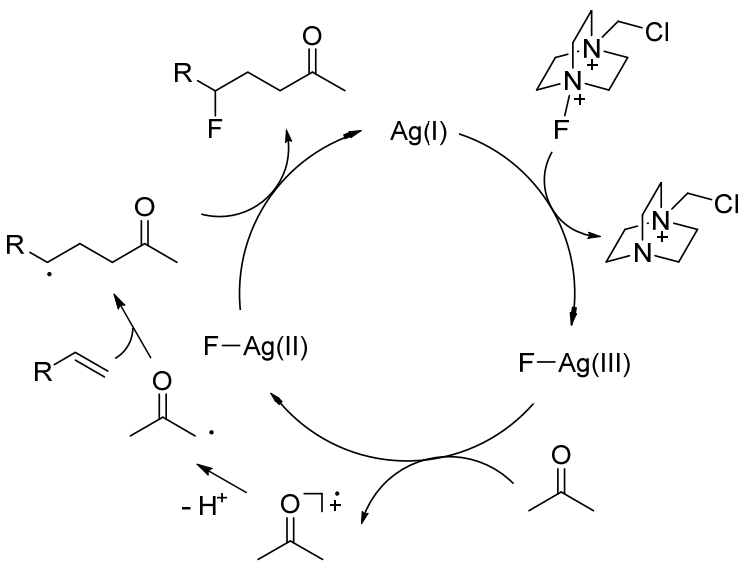

图式 5 可能的反应机理

Scheme 5 Possible mechanism

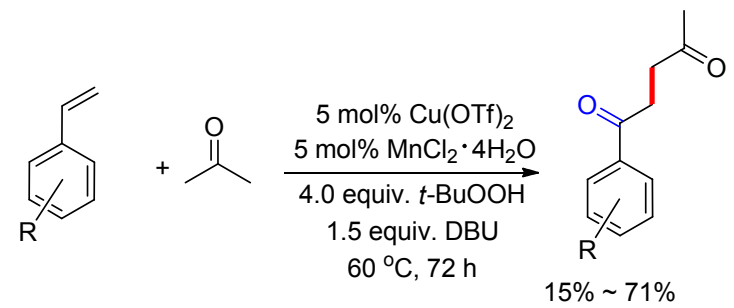

$\mathrm{Cao}$ 课题组 ${ }^{[23]}$ 报道了以分子氧为氧化剂, 利用铜催 化咪唑并 $[1,2-\alpha]$ 吡啶与羰基化合物(如 $N, N$-二甲基乙酰 胺和丙酮等)的反应，实现了 3-位碳一氢键的双羰基化反 应(Eq. 16). 他们只是提出了酰胺参与自由基反应的可 能机理，而并没有详细说明丙酮如何参与自由基的反 应. 

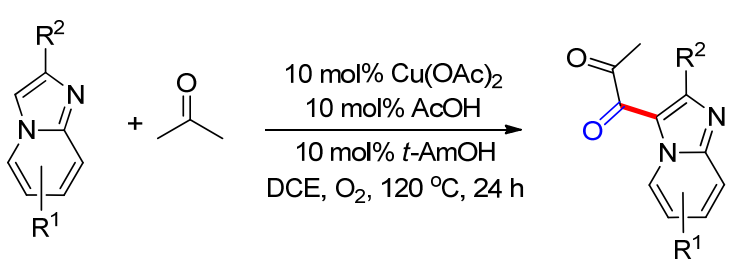

$15 \% \sim 84 \%$

最近, 我们课题组 ${ }^{[24]}$ 报道了以过氧叔丁醇及过氧 叔丁醚为混合氧化剂, 实现了二芳基二硫(硒)与丙酮的 氧化偶联, 高效地合成了一系列 $\alpha$-芳硫(硒)基丙酮化合 物(Eq. 17). 该反应官能团耐受性好, 产物产率较高. 我 们提出了产生丙酮自由基可能的反应历程(Scheme 6). 在过氧叔丁醇及过氧叔丁醚的混合氧化剂条件下, 产生 关键的羟基自由基 $\mathbf{A}$, 该自由基快速㩲取丙酮的 $\alpha$-氢生 成水, 同时产生丙酮自由基 $\mathbf{B}$, 再发生后续的自由基偶 联反应.
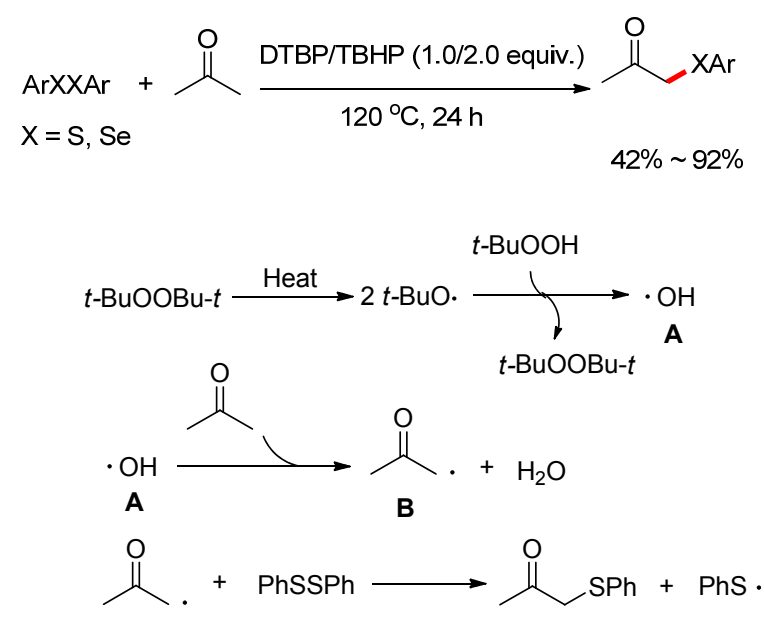

B<smiles>[B]C(=O)[C@H](C)CC(=O)C[SbH2]c1ccccc1</smiles>

图式 6 可能的反应机理

Scheme 6 Possible mechanism

受到这个反应的启发, 我们设想在该混合氧化剂体 系下，丙酮也能够发生自由基的碳一碳氧化偶联反应. 因此, 我们 ${ }^{[25]}$ 又报道了在该混合氧化体系下, 芳香醛与 丙酮的氧化偶联反应，合成了一系列的 1,3-二羰基化合 物(Eq. 18). 然而, 该反应需要加入催化量的对甲苯磺 酸, 以促进丙酮自由基 $\mathbf{B}$ 的生成. 另外, 该反应还需要 以四丁基碘化铵为催化剂, 以促进混合氧化剂产生过氧 叔丁基自由基 $\mathbf{C}$, 该自由基算取苯甲醛的氢生成过氧叔 丁醇, 同时产生苯甲酰基自由基 $\mathrm{D}$, 再发生后续的自由 基偶联得到目标产物(Scheme 7).

一般来说, 丙酮参与自由基偶联反应的条件相对比 较简单, 如光照、过氧化物或金属氧化物等, 有的反应

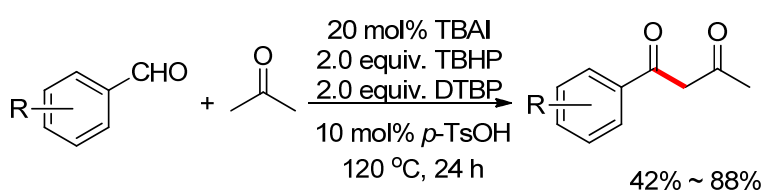

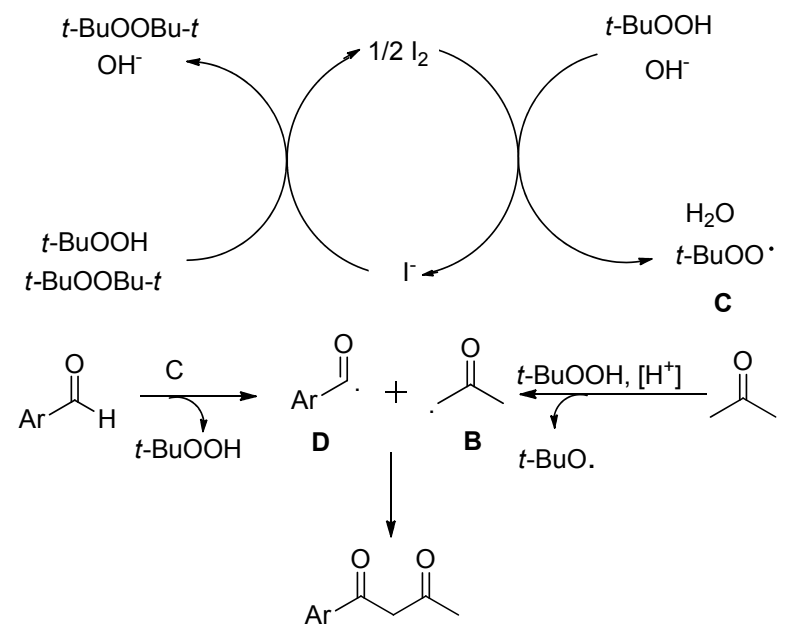

图式 7 可能的反应机理

Scheme 7 Possible mechanism

需要加入少量的有机酸促进丙酮自由基的产生.

\section{3 总结与展望}

综上所述，近年来有机化学工作者探索了丙酮参 与有机合成的新反应.一方面, 过渡金属催化丙酮与芳 香卤化物的交叉偶联，实现了羰基 $\alpha$-位的单芳基化反 应. 设计具有富电子且大位阻配体的催化体系对该类反 应非常关键. 探索更加温和的反应条件、催化剂及配体 的低用量以及更宽的底物适用范围也是今后努力的方 向. 另一方面, 丙酮参与自由基的氧化偶联反应, 合成 了各式各样的有机化合物. 然而, 大部分反应局限于丙 酮对烯烃的自由基加成. 探索丙酮参与不同类型底物的 自由基反应，构建碳一碳、碳一杂键是未来发展的方向.

\section{References}

[1] (a) Pan, J.; Wang, Y.; Chen, S.; Zhang, X.; Wang Y.; Zhou, Z. Tetrahedron 2016, 72, 240.

(b) Wang, F.; Liu, Y.; Qi, Z.; Dai, W.; Li, X. Tetrahedron Lett. 2014, 55,6399 .

(c) Lu, A.; Wu, R.; Wang, Y.; Wu, G.; Zhou, Z.; Fang, J.; Tang, C. J. Org. Chem. 2011, 76, 3872.

(d) Onodera, G.; Matsumoto, H.; Nishibayashi, Y.; Uemura, S. Organometallics 2005, 24, 5799.

(e) Özkar, S.; Finke, R. G. J. Am. Chem. Soc. 2005, 127, 4800.

(f) Noland, W. E.; Konkel, M. J.; Konkel, L. C.; Pearce, B. C. J. Org. Chem. 1996, 61, 451.

[2] For selected applications, see: (a) Miyaura, N.; Suzuki, A. Chem. Rev. 1995, 95, 2457.

(b) Ma, D.; Cai, Q. Acc. Chem. Res. 2008, 41, 1450.

(c) Hartwig, J. F. Acc. Chem. Res. 2008, 41, 1534. 
(d) Bellina, F.; Rossi, R. Chem. Rev. 2010, 110, 1082.

(e) Xiao, Q.; Zhang, Y.; Wang, J. Acc. Chem. Res. 2013, 46, 236.

[3] (a) Palucki, M.; Buchwald, S. L. J. Am. Chem. Soc. 1997, 119, 11108.

(b) Hamann, B. C.; Hartwig, J. F. J. Am. Chem. Soc. 1997, 119, 12382.

(c) Satoh, T.; Kawamura, Y.; Miura, M.; Nomura, M. Angew. Chem., Int. Ed. 1997, 36, 1740.

[4] (a) Culkin, D. A.; Hartwig, J. F. Acc. Chem. Res. 2003, 36, 234.

(b) Shao, Z.; Zhang, H. Chin. J. Org. Chem. 2005, 25, 282.

(c) Gong, J. F.; Xu, C.; Wu, Y. J. Prog. Chem. 2006, 18, 752.

[5] (a) Chobanian, H. R.; Liu, P.; Chioda, M. D.; Guo, Y.; Lin, L. S. Tetrahedron Lett. 2007, 48, 1213.

(b) Su, W. P.; Raders, S.; Verkade, J. G.; Liao, X. B.; Hartwig, J. F. Angew. Chem., Int. Ed. 2006, 45, 5852.

(c) Liu, P.; Lanza, Jr., T. J.; Jewell, J. P.; Jones, C. P.; Hagmann, W. K.; Lin, L. S. Tetrahedron Lett. 2003, 44, 8869.

(d) Kosugi, M.; Suzuki, M.; Hagiwara, I.; Goto, K.; Saitoh, K.; Migita, T. Chem. Lett. 1982, 939.

[6] Hesp, K. D.; Lundgren, R. J.; Stradiotto, M. J. Am. Chem. Soc. 2011, 133, 5194.

[7] Alsabeh, P. G.; Stradiotto, M. Angew. Chem., Int. Ed. 2013, 52, 1.

[8] Rotta-Loria, N. L.; Borzenko, A.; Alsabeh, P. G.; Lavery, C. B.; Stradiotto, M. Adv. Synth. Catal. 2015, 357, 100.

[9] Ackermann, L.; Mehta, V. P. Chem. Eur. J. 2012, 18, 10230.

[10] Li, P.; Lü, B.; Fu, C.; Ma, S. Adv. Synth. Catal. 2013, 355, 1255.

[11] (a) Cartney, D.; Guiry, J. P. Chem. Soc. Rev. 2011, 40, 5122. (b) Ana, R.; Pathak, T. P.; Sigman, M. Chem. Rev. 2011, 111, 1417.

[12] Gäbler, C.; Korb, M.; Schaarschmidt, D.; Hildebrandt, A.; Lang, H.; Adv. Synth. Catal. 2014, 356, 2979.

[13] (a) MacQueen, P. M.; Chisholm, A. J.; Hargreaves, B. K. V.; Stradiotto, M. Chem. Eur. J. 2015, 21, 11006. (b) Schranck, J.; Rotzler, J. Org. Process Res. Dev. 2015, 19, 1936.

[14] Fu, W. C.; So, C. M.; Chow, W. K.; Yuen, O. Y.; Kwong, F. Y. Org. Lett. 2015, 17, 4612.

[15] Schranck, J.; Tlili, A.; Alsabeh, P. G.; Neumann, H.; Stradiotto, M.; Beller, M. Chem. Eur. J. 2013, 19, 12624.

[16] For selective reviews, see: (a) Iqbal, J.; Bhatia, B.; Nayyar, N. K. Chem. Rev. 1994, 94, 519.

(b) Sibi, M. P.; Porter, N. A. Acc. Chem. Res. 1999, 32, 163.

(c) Gansäuer, A.; Bluhm, H. Chem. Rev. 2000, 100, 2771. (d) Robertson, J.; Pillai, J.; Lush, R. K. Chem. Soc. Rev. 2001, 30, 94.

(e) Studer, A. Chem. Soc. Rev. 2004, 33, 263.

(f) Brown, S. S.; Stutz, J. Chem. Soc. Rev. 2012, 41, 6405.

(g) zirakis, M. D.; Orfanopoulos, M. Chem. Rev. 2013, 113, 5262.

(h) Dénès, F.; Schiesser, C. H.; Renaud, P. Chem. Soc. Rev. 2013, 42,7900 .

[17] (a) Norrish, R. G.; Bond, C. H. Nature 1936, 138, 1016.

(b) Büchi, G.; Inman, C. G.; Lipinsky, E. S. J. Am. Chem. Soc. 1954, $76,4327$.

(c) Nau, W. M.; Cozens, F. L.; Scaiano, J. C. J. Am. Chem. Soc. 1996, 118, 2275.

(d) Reusch, W. J. Org. Chem. 1962, 27, 1882.

(e) Majerski, K. M.; Pavlović, D.; Kulyk, M. Š. J. Org. Chem. 1993, 58, 252.

(f) Chung, W.-S.; Ho, C.-C. Chem. Commun. 1997, 317.

(g) Toltl, N. P.; Leigh, W. J. Organometallics 1996, 15, 2554.

(h) Ghandi, K.; Addison-Jones, B.; Brodovitch, J.-C.; McCollum, B. M.; McKenzie, I.; Percival, P. W. J. Am. Chem. Soc. 2003, 125, 9594.

(i) Shiraishi, Y.; Tsukamoto, D.; Hirai, T. Org. Lett. 2008, 10, 3117.

(j) Tsukamoto, D.; Shiraishi, Y.; Hirai, T. J. Org. Chem. 2010, 75, 1450 .

[18] Schweitzer-Chaput, B.; Demaerel, B. J.; Engler, H.; Klussmann, M. Angew. Chem., Int. Ed. 2014, 53, 8737.

[19] (a) Xia, X.-F.; Zhu, S-L.; Zeng, M.; Gu, Z.; Wang, H.; Li, W. Tetrahedron 2015, 71, 6099.

(b) oess, E.; Karanestora1, S.; Bosnidou1, A.-E.; Schweitzer-haput, B.; Hasenbeck, M.; Klussmann, M. Synlett 2015, 1973.

[20] Chu, X.-Q.; Meng, H.; Zi, Y.; Xu, X.-P.; Ji, S.-J. Chem. Eur. J. 2014, 20, 17198

[21] Zhu, L.; Chen, H.; Wang, Z.; Li, C. Org. Chem. Front. 2014, 1, 1299.

[22] Lan, X.-W.; Wang, N.-X.; Zhang, W.; Wen, J.-L.; Bai, C.-B.; Xing, Y.; Li, Y.-H. Org. Lett. 2015, 17, 4460.

[23] Wang, C.; Lei, S.; Cao, H.; Qiu, S.; Liu, J.; Deng, H.; Yan, C. J. Org. Chem. 2015, 80, 12725.

[24] Yan, G.; Borah, A. J.; Wang, L.; Pan, Z.; Chen, C.; Shen, X.; Wu, X. Tetrahedron Lett. 2015, 56, 4305.

[25] Shen, X.; Borah, A. J.; Cao, X.; Pan, W.; Yan, G.; Wu, X. Tetrahedron Lett. 2015, 56, 6484. 